\title{
Effectiveness of disinfection with alcohol 70\% (w/v) of contaminated surfaces not previously cleaned
}

\author{
Maurício Uchikawa Graziano ${ }^{1}$ \\ Kazuko Uchikawa Graziano ${ }^{2}$ \\ Flávia Morais Gomes Pinto ${ }^{3}$ \\ Camila Quartim de Moraes Bruna ${ }^{3}$ \\ Rafael Queiroz de Souza ${ }^{4}$ \\ Cesar Angelo Lascala ${ }^{5}$
}

\begin{abstract}
Objective: To evaluate the disinfectant effectiveness of alcohol $70 \%(w / v)$ using friction, without previous cleaning, on work surfaces, as a concurrent disinfecting procedure in Health Services. Method: An experimental, randomized and single-blinded laboratory study was undertaken. The samples were enamelled surfaces, intentionally contaminated with Serratia marcescens microorganisms ATCC $1475610^{6} \mathrm{CFU} / \mathrm{mL}$ with $10 \%$ of human saliva added, and were submitted to the procedure of disinfection WITHOUT previous cleaning. The results were compared to disinfection preceded by cleaning. Results: There was a reduction of six logarithms of the initial microbial population, equal in the groups WITH and WITHOUT previous cleaning $(p=0.440)$ and a residual microbial load $\leq 10^{2} \mathrm{CFU}$. Conclusion: The research demonstrated the acceptability of the practice evaluated, bringing an important response to the area of health, in particular to Nursing, which most undertakes procedures of concurrent cleaning /disinfecting of these work surfaces.
\end{abstract}

Descriptors: Ethanol; 2-Propanol; Disinfection; Hospital Infection Control Program; Nursing.

\footnotetext{
${ }^{1}$ MSc, Dentist.

2 PhD, Full Professor, Escola de Enfermagem, Universidade de São Paulo, São Paulo, SP, Brazil.

3 Doctoral Students, Escola de Enfermagem, Universidade de São Paulo, São Paulo, SP, Brazil. Scholarship holder from Coordenação de Aperfeiçoamento de Pessoal de Nível Superior (CAPES).

${ }^{4}$ Doctoral Student, Escola de Enfermagem, Universidade de São Paulo, São Paulo, SP, Brazil. Scholarship holder from Conselho Nacional de Desenvolvimento Científico e Tecnológico (CNPq).

${ }^{5} \mathrm{PhD}$, Professor, Faculdade de Odontologia, Universidade de São Paulo, São Paulo, SP, Brazil.
} 


\section{Introduction}

Because the sanitization of the health professionals' hands - necessary to break the cycle of transmission of microorganisms from a reservoir to a susceptible host - may be neglected, possibly causing cross-infection related to care procedures, the microbial contamination of surfaces touched by professionals' hands must be eliminated by reliable methods. (1). The classic and agreed recommendation of the reliable methods for decontaminating such surfaces consists of cleaning the area prior to disinfecting it with a microbicidal agent such as alcohol $70 \%(w / v)^{(2)}$. This is the an intermediate-level germicide, according to the classification of the Centers for Disease Control and Prevention $(C D C)^{(3)}$, which is most available and used in the health care services (both for ethyl alcohol and 2-propanol), principally due to its low cost when compared with other products.

In care practice the direct application of alcohol on contaminated surfaces, without these being cleaned previously, is observed with relative frequency. This procedure would contradict, a priori, infection control good practices in health care services(2).

Disinfection is defined as "a process that eliminates many or all pathogenic microorganisms, except bacterial spores, on inanimate objects. In health-care settings, objects usually are disinfected by liquid chemicals or wet pasteurization"(3).

The classification of materials used in health care according to their potential risk for causing infections is well-defined as critical, semicritical and noncritical(4). The same cannot be affirmed for inert surfaces. There is a consensus among infection control professionals that surfaces touched by health professionals' hands should be minimally disinfected. Analyzing from a quantitative viewpoint, contaminations of the order of $10^{2-3}$ ColonyForming Units (CFU) are acceptable for noncritical products $^{(5)}$ defined as those which enter into contact with the patients' intact skin or those which do not enter into contact with them. By extension, this standardization may be extrapolated for surfaces which may be touched by the health professionals' hands during care activities, accepting, on the surface investigated, the presence of up to $10^{2-3}$ CFU as a maximum disinfected surface microbial load.

Considering the above, the present study's question was: is the disinfection with alcohol 70\% $(\mathrm{w} / \mathrm{v})$ of contaminated surfaces WITHOUT previous cleaning satisfactory? This procedure meets one of the requirements of disinfection, which is to reduce, at the minimum, five logarithms of the initial microbial inoculum(6) while at the same time the residual contamination may not pass the order of $10^{2-3} \mathrm{CFU}^{(5)}$.

The relevancy of the response to this question of the research was justified by the confirmation or refutation of the safety of a practice present in Brazilian health care environments.

\section{Material and Method}

The study design was experimental, laboratorybased, randomized and single-blinded.

The samples were constituted by enamelled surfaces $(21 \times 47.5 \mathrm{~cm})$, which had previously been exposed to the contaminant challenge, the test microorganism Serratia marcescens ATCC $1475610^{6}$ CFU/mL with 10\% of human saliva added. The following study groups were formed:

Experimental group: alcohol 70\% (w/v) was applied directly, with friction (in circular movements) for 30" WITHOUT prior cleaning of the intentionallycontaminated surfaces. This group reproduced a practice present in care activities in health-care.

Comparative control group: initially carried out a 'classic' cleaning with water and detergent under friction (using circular movements) followed by rinsing and consecutive disinfection through the application of alcohol $70 \%$ (w/v) for $30^{\prime \prime}$ on the intentionally-contaminated surfaces (idem in circular movements).

Positive control group: contaminated surfaces, with no treatment at all.

The Serratia marcescens selected as the contaminant challenge in the present investigation is an opportunist, Gram-negative microorganism. It was initially considered non-pathogenic and used to study forms of transmission between bacteria, due to the ease with which it may be identified because of its characteristic red pigmentation(7). The organic matter (human saliva) was added to the suspension of the test microorganism with the aim of further increasing the challenge in the evaluation of the techniques for decontaminating surfaces, challenging the assistential practice scene.

The size of the samples calculated for the experimental and comparative control groups, was 84 sample units each, for a significance of $5 \%$ and power of $80 \%{ }^{(8)}$. 
The experiments were conducted in the Microbiology Testing Laboratory of the Department of Medical-Surgical Nursing at the University of São Paulo School of Nursing (EE-USP).

One $\mathrm{mL}$ of the contaminant challenge was spread on the surface with the aid of a sterilized spatula. After this had dried at room temperature, randomization was undertaken, for the definition of each sample: whether it would belong to the experimental group or the comparative control group.

Hydrated ethyl alcohol $70 \%(\mathrm{w} / \mathrm{v})$, sold under the trademark ITAJÁ ${ }^{\circledR}$, was used, a product registered with the Brazilian Ministry of Health under no 324550003 as a hospital disinfectant for fixed surfaces.

Immediately after the complete evaporation of the alcohol from the surfaces, microbiological collection was carried out through friction with a sterilized swab, rubbing it along the length of the surface. Next, using aseptic technique and under laminar flow, the tip of the swab was broken off, submersed in a sterilized test tube with $1 \mathrm{~mL}$ of saline $0.9 \%$, and agitated in a Vortex ${ }^{\circledR}$ for one minute. Sequentially, the entire contents of the test tube were poured into the center of a sterilized Petri dish, and onto this was poured $20 \mathrm{~mL}$ of the TSA (Trypticase Soy Agar) medium produced by Difco ${ }^{\circledR}$, at the approximate temperature of $30^{\circ} \mathrm{C}$ (pour plate technique). The Petri dishes were incubated at $22^{\circ} \mathrm{C}$ for 14 days, with daily readings taken of the recovery of the Serratia marcescens and the recovered CFU counted. The final reading was taken by two researchers, one of whom was blinded as to whether the dish under evaluation belonged to the comparative control group or to the experimental group (single-blinded).

The positive control group was collected in triplicate at the start of each day of the experiments, shortly after the contamination of the surfaces, so as to confirm the presence of the microbial challenge.

The averages of the recovered CFU in the experimental group and the comparative control group were compared through the statistical Student $t$ test.

\section{Results}

The total number of dishes with growth versus the total number of dishes was 15/84 for the Experimental group, and 9/84 for the Comparative control group.

Table 1 shows the number of CFU of the recovered test microorganisms in both Groups.
Table 1 - Distribution of the CFU of Serratia marcescens ATCC 14756, in the samples from the experimental (application of alcohol 70\% w/v WITHOUT prior cleaning) and comparative control (WITH prior cleaning) groups. São Paulo, March 2011

\begin{tabular}{|c|c|c|c|}
\hline \multicolumn{2}{|c|}{$\begin{array}{l}\text { Growth in } \\
\text { Experimental group }\end{array}$} & \multicolumn{2}{|c|}{$\begin{array}{l}\text { Growth in } \\
\text { Comparative control group }\end{array}$} \\
\hline $\mathrm{N}^{\circ}$ of dish & CFU & $\mathrm{N}^{\circ}$ of dish & CFU \\
\hline 1 & 1 & 10 & 1 \\
\hline 6 & 1 & 12 & 1 \\
\hline 8 & 1 & 26 & 1 \\
\hline 10 & 1 & 29 & 1 \\
\hline 15 & 2 & 48 & 1 \\
\hline 16 & 1 & 53 & 1 \\
\hline 18 & 1 & 87 & 1 \\
\hline 32 & 1 & 85 & 3 \\
\hline 41 & 1 & 80 & 3 \\
\hline 44 & 1 & & \\
\hline 55 & 3 & & \\
\hline 80 & 1 & & \\
\hline 81 & 1 & & \\
\hline 83 & 1 & & \\
\hline 84 & 3 & & \\
\hline Total & 20 & & 13 \\
\hline
\end{tabular}

The descriptive statistics and the $p$ values comparing the Experimental group and the Comparative control group are presented in Figure 1.

\begin{tabular}{|l|c|c|}
\hline & $\begin{array}{c}\text { Experimental } \\
\text { group }\end{array}$ & $\begin{array}{c}\text { Comparative } \\
\text { control group }\end{array}$ \\
\hline Mean & $1.3 \mathrm{CFU}$ & $1.4 \mathrm{CFU}$ \\
\hline Standard deviation & 0.7 & 0.8 \\
\hline Minimum Value & $1 \mathrm{CFU}$ & $1 \mathrm{CFU}$ \\
\hline Maximum Value & $3 \mathrm{CFU}$ & $3 \mathrm{CFU}$ \\
\hline Median & $1 \mathrm{CFU}$ & $1 \mathrm{CFU}$ \\
\hline
\end{tabular}

$p=0.440$ (Student $t$ test)

Comparison between the groups of the two growth proportions $p=0.2703$

Figure 1 - Mean, standard deviation, minimum values and maximum values for recovered CFU of Serratia marcescens ATCC 14756 and the median and $p$ values comparing the Experimental and Comparative control groups. São Paulo, Brazil, March 2011

In the majority of the standardized methods for evaluating the effectiveness of chemical disinfectants, the decrease required in the initial inoculum is a minimum of 5 logarithms ${ }^{(6)}$. The reduction in the 
microorganisms in both the experimental group and the comparative control group was homogenous and marked, in the order of 6 logarithms (99.9999\%), and the $p$ value was $>0.05$ in the comparison of the averages of microbial reduction and in the proportion of growth between both groups (experimental group and comparative control group).

The growths of the test microorganism in the positive control dishes were all satisfactory after 24 hours of incubation at the temperature of $22^{\circ} \mathrm{C}$. Uncountable CFU were recovered in each dish, confirming the challenge imposed on the experimental and comparative control groups during the experiments.

\section{Discussion}

The results of the present investigation demonstrated the disinfectant effectiveness of alcohol $70 \%(\mathrm{w} / \mathrm{v})$ applied directly to contaminated surfaces, presenting results which were equivalent when compared to the classically-recommended method of decontamination, which consists of cleaning the surface prior to applying alcohol $70 \%(\mathrm{w} / \mathrm{v})$.

These findings bring an important theoretical framework to infection control in health-care facilities, as - explained among other reasons by the complexity involved in decontaminating surfaces in two stages (disinfection following cleaning) - the classical recommendation is not always followed by health professionals.

The health products industry, attent to health professionals' needs, has launched highly practical products on the market in the form of sprays and humidified wipes, based on fourth generation quaternary ammonium salts or other active disinfecting ingredients which, applied directly on contaminated surfaces, clean and disinfect the area simultaneously in a few seconds through the technique known as spray-wipe. In the dayto-day context of health care facilities in Brazil, however, the most available and used product is alcohol 70\% $(w / v)$, mainly due to its lower cost compared to these new products.

Two arguments have sustained the rebuttal of the practice of using alcohol $70 \%(w / v)$ directly on contaminated surfaces: the first is the inactivation of the alcohol $70 \%(\mathrm{w} / \mathrm{v})$ by the organic matter, and the second is that the alcohol $70 \%(\mathrm{w} / \mathrm{v})$ has properties which fix organic matter to surfaces where it is applied, which can, in theory, lead to the accumulation of organic matter, including microorganisms.
Concerning the first argument, research(9) on hand sanitization with alcohol formulas, in which blood was the organic matter challenge, refuted the hypothesis that this can inactivate the action of the alcohol $70 \%$ $(w / v)$. Although this experiment was not on an inert surface, it is possible to extrapolate the conclusion that alcohol promotes microbial reduction (also of Serratia marcescens) by at least $99.9 \%$ up to $99.99999 \%$, in the presence of blood as organic matter on the hands to be degermed, supporting the findings of the present investigation, which reduced the microbial load on the surface by the order of $99.9999 \%$ in the presence of the organic matter saliva.

As for the second argument, that alcohol $70 \%(w / v)$ has the properties of fixing organic matter on surfaces to which it is applied, no studies were found proving this. To the contrary of this affirmation, this chemical agent is known as an important solvent, as it contains in its chain $(\mathrm{CH} 3 \mathrm{CH} 2-\mathrm{OH})$ one polar part and one non-polar. The link with the hydroxyl is the polar part of the structure and the carbon chain is the non-polar part. Fats are nonpolar compounds, and are therefore soluble in non-polar compounds. Thus water, which is a polar compound, does not dissolve fats, while ethanol, which has polar and non-polar parts, dissolves fats and can also be dissolved in water(10). In the laboratory procedures with the experimental group, the alcohol was visibly a cleaning agent under visual inspection.

Research projects which investigated the disinfectant action of alcohol on contaminated surfaces, in the context of practices in health, and which did not include prior cleaning, arrived at satisfactory results supporting the present investigation's results ${ }^{(11-13)}$.

A Brazilian study(11) evaluated comparatively the germicidal action of four products on contaminated surfaces: ethyl alcohol at $77^{\circ} \mathrm{GL}$ (Parati ${ }^{\circledR}$ 92.8 $8^{\circ}$ Alc/ vol, $96^{\circ} \mathrm{GL}$ ), phenolic compound (Duplofen ${ }^{\circledR}$ ), iodophorPVP-I (L.M.Farma ${ }^{\circledR}$ ) and solution of ethyl alcohol at $77^{\circ} \mathrm{GL}$ with $5 \%$ of chlohexidine (Manipulário ${ }^{\circledR}$ ). Four areas in an odontological environment were chosen for data collection - odontological equipment, the surface of the hand basin, the dental chair's head-rest, and the frontal external surface of the reflector, after five minutes of functioning of the high speed handpieces, which is when one finds the worst case scenario of contamination of environmental surfaces. After the dry removal of the residue of organic matter such as blood, saliva and tissue, the areas were disinfected with the test products; the spray-wipe-spray technique was applied, which consisted of spraying the substance 
to be tested, rubbing the area with sterilized gauze using continuous movements in only one direction, and repeating the application of the germicide, spraying the solution again and leaving it in contact for five minutes. Samples were collected from each point, using contact plates containing selective culture media. The results were analyzed statistically using the Student $t$ test for comparing the averages of CFU/plate, before and after the disinfection. Ethyl alcohol at $77^{\circ} \mathrm{GL}$ presented a statistically-significant microbial reduction after the process of disinfection, despite not having been the most efficient of the four products tested.

In search of an answer to the best method of applying alcohol 70\% (w/v) for decontaminating surfaces, an English study ${ }^{(12)}$ published in 2009 investigated in vitro the effectiveness of two methods of applying alcohol $70 \%(\mathrm{w} / \mathrm{v}$ ) on surfaces deliberately contaminated with microorganisms, with $0.6 \%$ (w/v) of bovine serum albumin added. One of the methods tested was friction with alcohol $70 \%(\mathrm{w} / \mathrm{v})$ soaked in a cloth, for a period of contact of 10 seconds, and the other was the spray/dry wipe method. The microbial challenge contamination consisted of spores of Bacillus subtilis ATCC 6051, Staphylococcus epidermidis NCIMB 8853 and methicillin-resistant Staphylococcus aureus. As a result, the friction method using a cloth soaked with alcohol $70 \%(\mathrm{w} / \mathrm{v})$ performed better in reducing the microbial load than the spray/dry wipe method, endorsing the method used in the present investigation, and which reflects a common practice in the Brazilian health facilities.

Another Brazilian research project(13), concerned with the prevention of cross-infection mediated by the contamination of surfaces, studied the effectiveness of the surfaces' disinfection, testing aqueous solutions of chlorhexidine in the concentrations of $0.5 \%, 1 \%$, $2 \%, 3 \%$ and $4 \%$, comparing these with alcohol $70 \%$ $(w / v)$ in gel and liquid form. This study also included calculations related to their economic viability (search for greatest effectiveness of the diluted solutions). Strains of Streptococcus mutans, Staphylococcus aureus, Pseudomonas aeruginosa, Candida albicans and Klebsiella pneumoniae, at a density of $10^{8} \mathrm{CFU}$ were used as the challenge for the contamination of three different types of surface - leather, Formica, and stainless steel. After the intentional contamination, local disinfection was carried out using the spray-wipe-spray technique. After disinfection with each product, collections were made with contact plates (RODAC ${ }^{\circledR}$ ) containing agar BHI (Brain Heart Infusion Broth), followed by incubation and counting of CFU/plate. There was no recovery of strains of Klebsiella pneumoniae, Pseudomonas aeruginosa or Streptococcus mutans, in any of the surfaces or for any of the products tested, including the alcohol. Although there was recovery of the microorganism Staphylococcus aureus when the surface of the leather was disinfected with liquid alcohol, and when the surfaces of stainless steel and Formica were disinfected with alcohol gel, the reduction was significant, decreasing from $10^{8} \mathrm{CFU}$ of the initial microbial load to two CFU on the surface of the leather, two CFU on the surface of the stainless steel, and eight CFU on the surface of the Formica. There was also recovery of the microorganism Candida albicans on the surface of the stainless steel disinfected with the solution of chlorhexidine $0.5 \%$, the alcohol having shown itself to be effective. Despite the microbial recovery in the face of the action of the alcohol, the microbial reduction was around 7 logarithms, similar to the reduction found in the present investigation, which was of 6 logarithmic reductions, which attested to the effective disinfecting action.

The non-elimination of $100 \%$ of the challenge microorganisms in the disinfection with alcohol $70 \%$ $(w / v)$ may be attributed to the high concentration of the microbial inoculum used as the challenge which may have extrapolated the germicidal capacity of the products tested in the conditions of the experiments.

As noted in the introduction, analyzing from a quantitative perspective, contaminations in the order of $10^{2-3}$ are acceptable for noncritical products ${ }^{(5)}$, which enter into contact with intact skin, which standardization may be extrapolated for surfaces which may be touched by health professionals' hands during care activities. This being so, it is possible to deduce, based on the latest research analyzed, that an exaggeratedly contaminated surface, up to around $10^{8} \mathrm{CFU}$, would be decontaminated reliably using an alcohol $70 \%(\mathrm{w} / \mathrm{v})$ solution, applied directly with friction.

\section{Conclusion}

The present investigation demonstrated that there are no differences in the disinfectant effectiveness of alcohol $70 \%(\mathrm{w} / \mathrm{v})$ under friction, when applied WITH and WITHOUT prior cleaning on surfaces contaminated with a challenge (suspension of $10^{6} \mathrm{CFU}$ of Serratia marcescens ATCC 14756 with 10\% of human saliva added). Considering the consistency and plausibility of the method used, backed up by discussions of the evidence found in the literature, this research raised 
evidence of the absence of risk in the direct use of alcohol $70 \%(\mathrm{w} / \mathrm{v})$ for decontaminating contaminated surfaces, where health professionals' hands may be contaminated during care procedures.

\section{References}

1. Ferreira AM, Andrade D, Rigotti MA, Ferreira MVF. Condition of cleanliness of surfaces close to patients in an intensive care unit. Rev. Latino-Am. Enfermagem. 2011;19(3):557-64.

2. CDC-Center of Diseases Control and Prevention. Guideline for Environmental Infection Control in Health-Care Facilities: Recommendations of CDC and the Healthcare Infection Control Practices Advisory Committee (HICPAC). MMWR. [periódico na Internet]. 2003; [acesso 10 fev 2011]; 52(RR-10):1-48. Disponível em URL: http://www.cdc.gov/ncidod/hip/enviro/guide. htm.

3. Rutala WA, Weber DJ, HICPAC. Guideline for disinfection and sterilization in healthcare facilities, 2008. [acesso 10 fev 2011]; Atlanta, GA: US Department of Health and Human Services, CDC; 2008. Disponível em: http://www.cdc.gov/ncidod/dhqp/pdf/guidelines/ disinfection_nov_2008.pdf.

4. Spaulding EH. Chemical disinfection of medical and surgical materials. In: Lawrence CA, Block SS. Desinfection, Sterilization and Preservation. Philadelphia: Lea \& Febinger; 1968;517-31.

5. Gardener JF, Peel MM. Introduction to sterilization, disinfection and infection control. Melbourne: Churchill Livingstone; 1991.

6. Cremieux A, Fleurette J. Methods of testing disinfectants. In: Block SS. Disinfection, sterilization, and preservation. Philadelphia: Lea \& Febiger; 1991. p. 1009-27.

7. Eisenstein BI. Enterobacteriaceae. In: Mandell GLM, Douglas RG, Bennett JE. Principles and practice of infectous desease. Ed Churchill Livingstone; 1990.

8. Rosner B. Fundamentals of biostatistics. $6^{\mathrm{a}}$ ed. Chapter 10: Hypothesis testing: categorical date; 2006. p. 416.

9. Kawagoe JY, Graziano KU, Martino MDV, Siqueira I, Correa L. Bacterial reduction of alcohol-based liquid and gel products on hands soiled with blood. AM J Infect Control. 2011;39(9):785-7.

10. Silva LFGS. Caracterização da estabilização de eritrócitos por etanol [dissertação de mestrado]. Uberlândia (MG): Instituto de Genética e Bioquímica da Universidade Federal de Uberlândia; 2006. 53p.
11. Silva CRG, Jorge AOC. Avaliação de desinfetantes de superfície utilizados em Odontologia. Pesqui Odontol Bras. 2002;16(2):107-14.

12. Panousi1 MN, Williams GJ, Girdlestone S, Hiom SJ, Maillard JY. Evaluation of Alcohol Wipes Used During Aseptic Manufacturing. Society for Applied Microbiology. Letters Appl Microbiol. 2009;48:648-51.

13. Bambace AMJ, Barros EJA, Santos SSF, Jorge AOC. Eficácia de soluções aquosas de clorexidina para desinfecção de superfícies. Rev Biociênc. 2003;9(2):73-81. 